\title{
The Dermal Leishmaniases of Brazil, with Special Reference to the Eco-epidemiology of the Disease in Amazonia
}

\author{
R Lainson, J] Shaw, FT Silveira, AAA de Souza, RR Braga, EAY Ishikawa
}

Seção de Parasitologia, Instituto Evandro Chagas, Caixa Postal 691, 66017-970 Belém, PA, Brasil

Six species of Leishmania are at present known to cause cutaneous and/or mucocutaneous leishmaniasis in Brazil, and they are all to be found in the Amazon region of this country. The eco-epidemiology of each is discussed, with the observation that the Amazonian leishmaniases are all zoonoses, with their source in silvatic mammals and phlebotomine sandfly vectors. With man's destruction of the natural forest in southern Brazil, some sandfly species have survived by adapting to a peridomestic or domiciliary habitat in rural areas. Some domestic animals, such as dogs and equines are seemingly now involved in the epidemiology of the disease. No such process has yet been reported in the Amazon region, but may well take place with the continuing devastation of its forest.

Key words: Leishmania (Viannia) braziliensis - Leishmania (Viannia) guyanensis - Leishmania (Viannia) lainsoni - Leishmania (Viannia) shawi - Leishmania (Viannia) naiffi - Leishmania (Leishmania) amazonensis eco-epidemiology - Amazonia - Brazil

Until the late 1960 's, all forms of dermal leishmaniasis in Brazil were attributed to the single species Leishmania braziliensis. At that time, however, epidemiological studies were initiated at the Instituto Evandro Chagas, in Belém, State of Pará, entailing the examination of many patients and a wide variety of wild mammals and phlebotomine sandflies (Psychodidae: Phlebotominae). They soon indicated the presence of another species of Leishmania infecting man in the Amazon region of Brazil which, by virtue of its biological and biochemical resemblance to Leishmania mexicana, the causal agent of "chiclero's ulcer" in Central America, was given the subspecific name of Leishmania mexicana amazonensis Lainson \& Shaw, 1972.

Over the ensuing years, and largely as a result of these studies, it became clear that there existed, in fact, a multiplicity of different neotropical Leishmania species. Most of them have been shown to cause human dermal leishmaniasis in one form or another, with each showing its own peculiar ecological and epidemiological characteristics.

\section{MATERIALS AND METHODS}

Before discussing the eco-epidemiology of Amazonian dermal leishmaniasis it is appropriate to first outline the methods used in elucidating the "epidemiological triangle" of wild animal

Received 31 January 1994

Accepted 2 May 1994 reservoir - sandfly vector - man. More detailed accounts have previously been published elsewhere (Lainson 1982, Anon. 1984).

Human infection: detection and isolation of the parasite

Microscopic diagnosis - Clinical aspects can be deceiving, and differential diagnosis is required for dermal lesions due to a variety of other aetiological agents. There is no more sure diagnosis than seeing and isolating the parasite, but in inexperienced hands, or in poor laboratory conditions, the detection of amastigotes in stained smears may be sadly inefficient. Badly prepared smears, containing a host of contaminative bacteria, fungal spores, dust particles and staining deposit, may result in the failure to find Leishmania or, even worse, the registration of a false positive. Points to remember are the use of ultraclean and dry slides, avoidance of the necrosed central part of ulcers, adequate cleansing of the skin surface before making a biopsy or scraping from the margin of the lesion, avoidance of excessive blood in the smear, and rapid drying of thin smears for immediate fixation in absolute methanol which has not been allowed to absorb atmospheric moisture. Giemsa stain is that most widely used, but the quality of staining may vary with the brand: buffered distilled water should be at $7.0-7.6 \mathrm{pH}$, and free of algal contaminants. Placing the slides face down in flat staining dishes, rather than upright in Coplin jars, will avoid staining deposit if the surface scum is pushed to one side. 
In vitro culture - Parasites may be so scanty, especially in old lesions, that they defy detection in stained smears. Diagnosis will now depend on the culture of tissue juice or biopsied skin fragments from the border of the lesion in a suitable blood-agar medium, and the inoculation of such material into laboratory animals. Isolation of the parasite for subsequent identification is of utmost importance in eco-epidemiological studies: the use of monoclonal antibodies and DNA analysis for immediate identity of Leishmania species on glass slides or other substrates may hold promise for the future, but these sophisticated techniques are still not fully adapted to most field conditions and beyond the means of many laboratories in developing countries.

Growth of different Leishmania species may vary greatly in different culture media, and workers must ascertain those which are most suitable for the parasites likely to be encountered in their study areas.

Isolation in laboratory animals - The Syrian hamster remains the animal of choice: the mouse is much less susceptible to some leishmanias, particularly to those of the braziliensis complex. Aspirated tissue juice or a saline triturate of biopsied tissue is usually inoculated intradermally into the skin of the nose or feet, and the animals examined periodically for the appearance of skin lesions. Some Leishmania species will produce an inapparent skin infection, or one which is of a transient nature. For this reason at least two hamsters should be inoculated, so that cultures may be made from the inoculation-site of one after a few weeks, while the other(s) are retained. A year or more may elapse before some parasites produce a visible skin lesion.

The intradermal ("Montenegro") skin-test This widely used skin-test is highly specific and of great use in quantitative epidemiology: In diagnosis, however, the following points must be borne in mind: (a) a positive reaction may be due to a past infection and have nothing to do with the present lesion of the patient; (b) positive reactions may be registered in persons with no present or past evidence of infection, and (c) a small percentage of parasitologically positive patients may produce no reaction. The test is regularly negative in cases of diffuse, anergic leishmaniasis.

Identification, description and preservation of Leishmania isolates

Correct identification of the parasite by recognized biological and biochemical criteria (Lainson \& Shaw 1987) is imperative in ecoepidemiological studies, especially where a number of different leishmanias are circulating in nature. The original code of the laboratory in which an isolate is made should always be incorporated into the descriptive labelling of that parasite, using the coding method recommended by the World Health Organization (Anon. 1984). Strains should be preserved in liquid $\mathrm{N}_{2}$ as soon as possible after isolation, and deposited in one or more international reference centres.

\section{Pin-pointing the sandfly vector}

This may be relatively simple in regions where there are few sandfly species, but is clearly more difficult in areas where there are large numbers of anthropophilic species - as in the Amazon rain-forest. In our study area on the Serra dos Carajás, State of Pará, for example, we have registered 25 species of sandflies known to bite man! Logical steps in determining which of these are vectors in the transmission of the various Leishmania species to man are as follows: (a) accurately pin-pointing the exact area of transmission by carefully questioning patients, (b) establishing the time of year when transmission is at its peak, and (c) indicating all the anthropophilic species of sandflies in the area. Initially this will necessitate human bait, but once man-biters are recognized alternative methods should be used, including such well-tried devices as the Shannon and C.D.C. light-traps. When the habits of the suspected vectors are known they may be collected from their resting-sites, such as tree-trunks and animal burrows.

The final process of incriminating the vector of each Leishmania species in the area will depend on detecting, isolating and identifying the parasites of infected sandflies during mass-dissections of the captured insects. When the study area is far from home-base this, and the inoculation of the flagellates into hamsters and culture tubes, must be done in the field.

With luck, infected flies may be encountered during the initial dissections made to establish the composition of the local sandfly population (it is, incidentally, easier to recognise the species of a freshly dissected female sandfly than it is to identify specimens fixed in alcohol). If, however, this is done when the population is at its peak the chances of finding infected flies are slim, because a high proportion of the sandfly catch will be newly hatched, nulliparous females. Much higher infection-rates are found when the population is declining, at the onset of dryer weather.

One swallow does not make a summer, and one infected fly does not conclusively indicate a vector. Dissections and isolations must continue, to find a significant number of infections in a given sandfly species and to demonstrate heavy. promastigote infection in the anterior part of the fly's intestine. Concluding evidence will be obtained by indicating an intimate reservoirhost/sandfly/man contact, and by experimentally 
transmitting the parasite by the bite of the suspected vector species.

Incrimination of the wild mammalian reservoirhost(s)

Entomological data on the biting habits and preferential hosts of the sandfly vector will give clues as to the most likely wild mammalian hosts: canopy dwellers and sandflies resting on tree trunks are likely to derive their infection from arboreal animals, and a vector found only at ground level clearly indicates a terrestrial reservoir. Analysis of fresh bloodmeals from sandflies is potentially useful, but must be interpreted with care because many of these insects have very catholic feeding habits. Finally, the screening of wild animals should always include the examination of both skin and viscera, and it should be remembered that most Leishmania species produce a benign, inapparent infection in their natural mammalian hosts. Skin-snips from the ears, nose, tail and feet are triturated in saline and inoculated intradermally into one pair of hamsters, and a similar suspension of liver and spleen intradermally into two others. Culture of skin and viscera fragments follows the same procedure as that used for the isolation of Leishmania from man.

\section{Quantitative studies}

These should accompany the qualitative studies, to provide information on the prevalence and incidence of the human disease due to the different Leishmania species of a given locality, and the ecology and population fluctuations of both the sandfly vectors and the reservoir-hosts all of which are a basic necessity before any control measures can even be contemplated.

\section{RESULTS}

To date we have indicated the presence of six species of Leishmania causing human cutaneous leishmaniasis in the Amazon region of Brazil. In recent publications (Lainson \& Shaw 1987, 1992) we have allocated five of these to the subgenus Viannia Lainson and Shaw 1987, and one to the subgenus Leishmania Saf'janova 1982, as follows:

Leishmania (Viannia) braziliensis Vianna, 1911 Leishmania (Viannia) guyanensis Floch, 1954 Leishmania (Viannia) lainsoni Silveira et al. 1987

Leishmania (Viannia) shawi Lainson et al. 1989 Leishmania (Viannia) naiffi Lainson and Shaw, 1989

Leishmania (Leishmania) amazonensis Lainson and Shaw, 1972
A seventh species, Leishmania (Leishmania) deanei Lainson and Shaw, 1977, of the porcupine Coendou, has yet to be found infecting man.

\section{Leishmania (V.) braziliensis}

Geographical distribution: what appears to be the same parasite has been reported from the States of Bahia, Ceará, Espirito Santo, Minas Gerais, Pará, Paraná, Rio de Janeiro, Rondônia, São Paulo and southern Amazonas. The exact distribution of the parasite remains doubtful, however, due to past inadequate methods of identification.

Known mammalian hosts: man - among wild animals, $L_{.}(V$.$) braziliensis sensu lato has been$ recorded from the rodents Proechimys, Rhipidomys, Oryzomys, Akodon and Rattus, and the marsupial Didelphis. In domestic animals infections have been registered in dogs and equines, principally in the extensively deforested areas of southeast and northeast Brazil.

Recorded sandfly hosts: in the primary forest of Serra dos Carajás, Pará, the principal vector is undoubtedly Psychodopygus wellcomei. This is a highly anthropophilic fly, biting man avidly at night and, in overcast weather, during the day. It is active only during the rainy season (approximately November to April), and enters into diapause during the dry season. In southeast Brazil, in the States of Rio de Janeiro and São Paulo, the vector is most probably Lutzomyia intermedia, with possible involvement of $L u$. migonei and $L u$. pessoai in São Paulo. In Minas Gerais and some northeastern States (Bahia and Cearả), Lu. whitmani has been incriminated. Contrary to Ps. wellcomei, the latter species have adapted to a peridomestic or even intradomiciliary habitat, doubtless as a result of man's destruction of their natural silvatic habitat.

Defining the eco-epidemiology of $L .(V$. braziliensis is at present rendered most difficult by the occurrence of what appears to be the same parasite in areas of very different ecology, and where the sandfly fauna is quite different. Ps. wellcomei, for example, is absent in many lowland areas of Pará, yet many isolates of Leishmania from cases of cutaneous and/or mucocutaneous leishmaniasis in these regions appear to be $L .(V$.$) braziliensis (or very similar$ variants or serodemes, as indicated by isoenzyme and monoclonal antibody identification). Furthermore, parasites recorded as " $L$. braziliensis", " $L$. braziliensis braziliensis" or "L. braziliensis sensu lato" are reported in geographic regions as far apart as Belize in Central America, and the State of São Paulo, Brazil! Clearly, the sandfly and mammalian faunas and the ecology of such regions are very different, and we are faced with the choice of one of two explanations: either $L$ (V.) braziliensis is exceptional in having a wide 
range of totally unrelated sandfly vector species, or we are dealing with an extensive complex of related species and subspecies, for which our present methods of separation are inadequate.

\section{Leishmania (V.) guyanensis}

Geographical distribution: the Amazon basin, north of the river Amazonas, in Amapá, north Pará and Amazonas, possibly extending into Roraima: in neighbouring countries, Guyana, Surinam, French Guyana and Colombia. Rare cases are registered from Pará, south of the river Amazonas.

Known mammalian hosts: man - the principal wild mammalian hosts are the two-toed sloth, Choloepus didactylus and the lesser anteater, Tamandua tetradactyla. Secondary hosts are found in marsupials (Didelphis) and, more rarely, rodents (Proechimys).

Recorded sandfly hosts: the major vector is Lu. umbratilis, a forest canopy and tree-trunk dwelling sandfly. This insect is found, often in enormous numbers, resting on the trunks of the larger trees, in primary forest, from where it readily attacks man when disturbed. Transmission of $L$. (V.) guyanensis to man is, therefore, principally during the daylight hours, especially in the early morning, and men engaged in cutting and clearing primary forest are at particularly high risk. As sloths are rather sedentary animals, tending to stay in a given spot for some time, the infection-rate of Lu. umbratilis on a tree harbouring an infected animal may reach very high proportions. The simultaneous bite of numerous infected flies, disturbed from their resting-site, is the most likely explanation for the frequency of multiple lesions in cases of leishmaniasis due to L. (V.) guyanensis.

Occasional infections have been recorded in Lu. anduzei, which is also a canopy and treetrunk dweller and perhaps has some role as a secondary vector. Infections reported in $L u$. whitmani sensu lato (Lainson et al. 1981) were probably those of $L(V$.$) shawi. (There is now$ strong evidence that " $L u$. whitmani" from Para is different from the type material of this species from Bahia).

The enzootic of $L(V$.$) guyanensis, as seen in$ primary, forest is unlikely to survive in secondary forest or man-made plantations, where there are few or no large trees. The microhabitat on trees of small girth is unfavourable for the sandfly vector, doubtless due to low humidity and the smooth surface of the small tree-trunks. In addition, such trees are not a favourable environment for a large and relatively heavy animal such as the sloth. In plantations of pine, gmelina and eucalyptus, this animal is deprived of its normal diet of foliage and fruits of native trees.
A high prevalence of cutaneous leishmaniasis due to $L(V$.$) guyanensis may be associated with$ human habitations in or very near to primary forest, leading to the impression that the sandfly vector has adapted to a peridomestic or domiciliary habitat (Arias \& Naiff 1981). There is no evidence as yet, however, that $L u$. umbratilis is able to dispense with its arboreal life, and such cases of leishmaniasis are almost certainly the result of infected sandflies being attracted to human dwelling places from the neighbouring forest, presumably by the lights of houses at night. Clearing the forest to a distance of about $400 \mathrm{~m}$ around a village surrounded by primary forest, in French Guyana, was found to entirely eliminate peridomestic transmission to man (Le Pont \& Pajot 1981).

Finally, although infection of the marsupial Didelphis has been found to be very rare or absent in primary forest where there is intensive transmission of $L(V$.$) guyanensis (Arias et al.$ 1981, Lainson et al. 1981), a high prevalence has been found in those captured in the peridomestic habitat associated with nearby primary forest (Arias \& Naiff 1981). Reasons for this are uncertain: neither is it clear as to whether or not these infected opossums serve as an effective source of parasites for sandflies, or if they merely represent dead-end, accidental hosts.

\section{Leishmania (V.) lainsoni}

Geographical distribution: until now this parasite has been recorded only from the State of Pará, Brazil, but it doubtless exists in other regions where the sandfly vector and reservoirhost occur together.

Recorded mammalian hosts: man - among wild animals, it has so far been found only in the "paca", Agouti paca (Rodentia: Dasyproctidae).

Known sandfly host: Lutzomyia ubiquitalis.

Steps in unravelling the eco-epidemiology of $L$. (V.) lainsoni have made an interesting story, commencing in 1981 when we isolated a Leishmania from a case of cutaneous leishmaniasis acquired in forest near the Pirelli rubber plantation, about $20 \mathrm{~km}$ from Belém, and referred to it simply as an "unnamed parasite of the subgenus Viannia" (Lainson \& Shaw 1987).

In 1983 we isolated another Leishmania from a single specimen of the sandfly $L u$. ubiquitalis, captured in forest in the foothills of the Serra dos Carajás, Pará, and placed it in the same category.

One year later, working on the doomed Island of Tocantins, destined to be submerged beneath the Tucurui Reservoir formed by the new hydroelectric dam, we made isolates from numerous specimens of $A$. paca. Once again, the parasite was considered merely as an unidentified member of the subgenus: Viannia (unpublished observations). 
Later on, numerous isolates of a Leishmania made from patients coming to the Instituto Evandro Chagas had aroused our interest by the peculiar morphology of their amastigotes and promastigotes and, following the observation that the parasite's isoenzyme profiles distinguished it from all other known species of Leishmania, the organisms was named $L .(V$.$) lainsoni (Silveira et$ al. 1987).

A major break-through came in 1990, when we investigated an area of forest where one of our patients had acquired his infection with $L$ (V.) lainsoni: among our sandfly catches we found eight specimens of $L u$. ubiquitalis heavily infected with this parasite (Silveira et al. 1991b). The jigsaw puzzle was finally to become a definite picture, however, when we compared the isoenzyme profiles of all our old and new isolates of Leishmania from Lu. ubiquitalis and A. paca with our type species of $L$. $(V$.$) lainsoni from$ man, and found them to be indistinguishable (Silveira et al. 1991a,b).

Seemingly, then, we had completed our ecoepidemiological study of this parasite - but for one puzzling feature that remained to be explained. We had never recorded $L u$. ubiquitalis biting man in the forest and, we were forced to admit, therefore lacked the most crucial evidence in incriminating any arthropod vector of a human disease! Consequently, we began a study of the behaviour of $\mathbf{L u}$. ubiquitalis, both in the laboratory and in the forest.

In the first place, we found that this sandfly would feed on man, quite avidly, when brought from the forest and maintained in the laboratory for some hours. This suggested, then, that this fly must also bite man in the forest, under certain conditions - which we had yet to define. Finally, any doubts of this were removed when a fully engorged $L u$. ubiquitalis was eventually taken while biting the arm of a man who was, appropriately enough, standing in the same spot in the forest where we caught our first infected $\mathbf{L u}$. ubiquitalis, nearly ten years previously (Lainson et al. 1992).

Clearly, however, this sandfly is not particularly fond of human blood, which explains the relatively low rate of infection with $L(V$. lainsoni in man, compared with that of $L(V$. braziliensis and $L(V$.$) guyanensis, both of which$ have highly anthropophilic sandfly vectors.

\section{Leishmania (V.) shawi}

Geographic distribution: at present, only known from Pará, Brazil.

Recorded mammalian hosts: man - reservoirhosts among the forest animals include monkeys (Cebus apella and Chiropotes satanas), sloths (Choloepus didactylus and Bradypus tridactylus) and the coatimundi (Nasua nasua). It is quite likely that other arboreal animals may be involved.

Recorded sandfly hosts: Lutzomyia whitmani s.l. (There is now strong evidence that " $L u$. whitmani" from Pará is different from the type material of this species from Bahia).

In 1948 Leonidas Deane noted the presence of amastigotes in spleen and liver smears from a two-toed sloth, $C$. didactylus captured in Abaetetuba, Pará. He remained uncertain, however, as to whether they belonged to a Leishmania species or represented a stage in the life-cycle of Endotrypanum (an intra-erythrocytic flagellate of sloths).

What was probably the same parasite was later isolated from the viscera of $C$. didactylus, from Acará, Pará, by Lainson and Shaw (1972). This time, however, there was no doubt regarding the organism's inclusion in the genus Leishmania, as it was studied in both hamsters and blood-agar cultures. It was considered to "...differ from $L$ b. braziliensis..." and to be "...closest to L. b. guyanensis..." (Lainson \& Shaw 1979, 1987).

In 1988 we recorded similar infections in the skin of C. apella and Ch. satanas (Cebidae), the viscera of a three-toed sloth, B. tridactylus, and the skin of a coatimundi, $N$. nasua, all from primary forest in the Serra dos Carajás, Pará. A comparison with $L$. (V.) braziliensis, $L$. (V.) guyanensis and $L$ (V.) panamensis, using monoclonal antibodies and isoenzyme profiles, led us to the conclusion that the organism was "...a hitherto undescribed parasite of the braziliensis complex", and it was given the name of $L(V$.$) shawi (Lainson et al. 1989).$

Incrimination of the sandfly $\mathrm{Lu}$. whitmani s.l. followed after the biological and biochemical characterization of 14 heavily infected flies. Two of these were caught in a C.D.C light-trap baited with a caged $C$. apella, and two in a Shannon trap, all in the same study area in which the infected animals were obtained. The remaining ten infections were in specimens of Lu. whitmani s.1. caught in light-traps or with human bait, in another of our work areas in Tucurui, Pará.

It was clearly now important to complete the third point of our epidemiological triangle - did $L$ (V.) shawi infect man? Accordingly, we took from our cryobank some isolates of Leishmania made from man, and which we had previously found to be similar to, but not identical with, $L$. $(V$.$) guyanensis. The isoenzyme profiles of$ eighteen of these showed twelve to be indistinguishable from the type strain of $L_{.}(V$.$) shawi:$ very slight differences in the PEP profiles of the other six led us to regard these merely as isoenzyme variants of the same parasite (Shaw et al. 1991). 
The steps leading up to the elucidation of the eco-epidemiology of $L(V$.$) lainsoni and L(V$. shawi clearly emphasise the enormous importance of religiously cryopreserving all isolates of Leishmania from sandflies, wild or domestic animals and man.

\section{Leishmania (V.) naiffi}

Geographic distribution: this has been poorly studied, but till now isolates appear to be limited to the States of Pará and Amazonas.

Recorded mammalian hosts: man - among wild animals the parasite is known only from the nine-banded armadillo, Dasypus novemcinctus.

Known sandfly hosts: Psychodopygus paraensis, Ps. ayrozai and Ps. squamiventris have all occasionally been found infected. Which of these is the principal vector, however, remains doubtful.

The nine-banded armadillo for long enjoyed a folkloric reputation as the fundamental source of cutaneous leishmaniasis in the Amazon region. The reasons for this are obscure, but probably have something to do with the frequent presence of phlebotomine sandflies in their burrows. The Tupi indian name for the armadillo is "tatu", and that for the sandfly, "tatuquira" - literally, the "armadillo-fly". Unfortunately for this hypothesis the sandfly species living in armadillo holes are not those which commonly bite man, and the case against $D$. novemcinctus remained somewhat thin.

In 1979, however, we added some substance to the myth when we isolated a Leishmania in blood-agar cultures made from the liver and spleen of an armadillo obtained from primary forest in Monte Dourado, north Pará (Lainson et al.1979).

In spite of the unique biological and biochemical nature of the parasite it did not receive its name until ten years later (Lainson \& Shaw 1989), and there still remained no firm evidence that it was the cause of human cutaneous leishmaniasis. In that year, however, Menezes et al. (1989) did record the isolation of a parasite from the skin lesion of a man coming from the Vale do Rio Trombetas region in Pará, which had an isoenzyme profile "...similar to the group of seven zymodemes of isolates from man, armadillos and sandflies from the Amazon region...". They gave no details of these, however, and described the organism as having round, vacuolated amastigotes which were "...larger than any recognized species of the subgenus Viannia". This is not at all suggestive of $L$ (V.) naiffi, which has amastigotes which are considerably smaller than most members of the subgenus.

Naiff et al. (1989) registered the presence of $L$ (V.) naiffi in D. novemcinctus from the State of
Amazonas, and what appeared to be the same parasite from a human skin lesion, based on biological characters of the organism.

Finally, Lainson et al. (1990) confirmed that the parasite recorded from man by Naiff et al. (1989) was indeed $L .(V$.$) naiffi, and registered$ another human infection with this parasite after comparison of the isolates with the type strain from $D$. novemcinctus, by way of monoclonal antibodies and isoenzyme profiles.

$L$. (V.) naiffi rarely produces a discernable skin lesion in the hamster, and we feel that for this reason human infection with this organism may frequently have been missed in the past, when inoculation of this animal with lesion-biopsy material has been the sole method of isolation attempted.

Nonetheless, judging from our isolates of Leishmania from man in Pará during the past 25 years, $L .(V$.$) naiffi does not appear to be a very$ important pathogen of man. Either because the major sandfly vector is not very anthropophilic (Ps. ayrozai?), or because the parasite commonly produces only an occult, benign infection in the skin of man, as it does in the hamster.

\section{Leishmania (L) amazonensis}

Geographic distribution: typically the Amazon basin, but rare records exist from the States of Bahia and Paraná.

Known mammalian hosts: man - the most important wild animal hosts are rodents (particularly species of Proechimys and Oryzomys), but the parasite is also commonly found in marsupials (Didelphis, Philander, Marmosa, Caluromys and Metachirus). We have recorded a single isolate from the fox, Cerdocyon thous.

Recorded sandfly hosts: the principal vector is Lu. flaviscutellata. Infections have less frequently been found in $\mathrm{Lu}$. olmeca nociva, a very closely related fly of the olmeca complex, to which $L u$. flaviscutellata belongs: it may play a secondary role within the enzootic.

$L$ ( $L$. amazonensis is extremely common in Amazonia, in a wide range of terrestrial animals. Furthermore, it occurs in all manner of woodland, including primary high forest, varzea, swamp forest, and any low, dense secondary growth resulting from deforestation. The enzootic has even spread into man-made forests such as plantations of pine and gmelina, due to the ready adaptation of rodents, marsupials and Lu. flaviscutellata to such habitats.

It is fortunate, indeed, that the sandfly vector is not greatly attracted to man. If it were, the problem of human cutaneous leishmaniasis in Amazonia would be very much greater than it already is: not only in terms of sheer numbers of cases, but due to the fact that $L\left(L_{0}\right)$ amazonensis is the cause of "diffuse, anergic cutaneous leish- 
maniasis" in individuals with a faulty cellmediated immunological system. This disease is not only highly mutilating, but also incurable by our present methods of treatment.

\section{DISCUSSION}

From this brief account of the Leishmania species known to cause dermal leishmaniasis in Pará and other parts of Amazonian Brazil, we see that these diseases are essentially zoonoses, with reservoirs in a wide variety of silvatic mammals, among which they are transmitted by different species of phlebotomine sandflies. Transmission is by no means an haphazard, indiscriminate affair, however: there exist distinct associations between certain mammalian and sandfly species, in specific ecological niches, and governed by certain environmental factors.

The number of Leishmania species or subspecies circulating in the Amazonian fauna is anybody's guess. Certainly we have but scratched the surface, and many more than we have discussed here will be discovered in the future. Tables I and II list mammals and sandflies in which we have recorded unidentified leishmanial parasites, or promastigotes which are most probably those of Leishmania. We either failed to isolate these organisms in blood-agar culture and hamsters, or the isolates were lost before they could be characterised.

In Amazonia, the cutaneous leishmaniases can be regarded as occupational diseases, contracted principally by forest-workers engaged in such activities as the construction of highways, hydroelectric dams, or other projects requiring the penetration and cutting of native forest. At risk, on a smaller scale, are topographers, geologists, botanists, zoologists, hunters, - and the occasional tourist!

This predominantly silvatic ecology of the different Leishmania species was doubtless the situation throughout Brazil and most other parts of Latin America before the Iberian colonization, and the relentless deforestation that followed.

\section{TABLE I}

Amazonian mammals found with Leishmania of doubtful taxonomy

\begin{tabular}{ll}
\hline Rodentia & $\begin{array}{l}\text { Rhipidomys leucodactylus } \\
\text { Proechimys spp. } \\
\text { Rattus rattus }\end{array}$ \\
& $\begin{array}{l}\text { Didelphis marsupialis } \\
\text { Mhilander opossum }\end{array}$ \\
& $\begin{array}{l}\text { Tamandua tetradactyla } \\
\text { Xenarthra }\end{array}$ \\
Primates & Homo sapiens \\
Chiroptera & An unidentified bat
\end{tabular}

\section{TABLE II}

Amazonian phlebotomine sandflies found with Leishmania or promastigotes of doubtful taxonomy

\begin{tabular}{ll}
\hline Psychodopygus amazonensis & Lutzomyia anduzei \\
Ps. chagasi & Lu. antunesi \\
Ps. paraensis & Lu. dendrophila \\
Ps. s. squamiventris & Lu. furcata \\
Ps. s. maripaensis & Lu. gomezi \\
Ps. wellcomei & Lu. h. hirsuta \\
& Lu. shawi \\
& Lu. tuberculata \\
& Lu. yuilli \\
\hline
\end{tabular}

Today, in Bahia, Minas Gerais, Rio de Janeiro, São Paulo and other affected regions, some sandfly species survived by adapting to a peridomestic or even intradomiciliary habitat in rural areas, and their reservoir of infection may now also include some domestic animals such as dogs and equines, which are frequently found with leishmanial skin lesions. It is very likely, however, that the primitive source of the parasite still lurks in the degraded forest remnants, which still harbour a variety of rodents and marsupials. The predominant surviving leishmanial parasite is $L$. (V.) braziliensis s.l. Many other Leishmania species were doubtless doomed to extinction together with their sandfly vectors, and it remains to be seen if this same process will ultimately be repeated in Amazonia, following man's continued devastation of its great forest (Fig.).

\section{ACKNOWLEDGEMENTS}

To the Wellcome Trust, London, for their unstinted support during 27 years of research on leishmaniasis in Amazonia by the Wellcome Parasitology Unit (19651992). Also to the Instituto Evandro Chagas, Fundação Nacional de Saúde, whose collaboration made these studies possible.

\section{REFERENCES}

Anon. 1984. The leishmaniases: report of a WHO expert committee. Tech Rpt Series 701: WHO, Geneva, 1984, 140 pp.

Arias JR, Naiff RD 1981. The principal reservoir host of cutaneous leishmaniasis in the Urban areas of Manaus, Central Amazon of Brazil. Mem Inst Oswaldo Cruz 76: 279-286.

Arias JR, Naiff RD, Miles MA, Souza AA 1981. The opossum, Didelphis marsupialis (Marsupialia: Didelphidae), as a reservoir host of Leishmania braziliensis guyanensis in the Amazon Basin of Brazil. Trans R Soc Trop Med Hyg 75: 537-541.

Deane LM 1948. Abstract of discussion on CA Hoare's paper The relationship of the haemoflagellates. Proc 4th Int Congr Trop Med Malar, Washington DC, 10-18 May.

Floch H 1954. Leishmania tropica guyanensis n.ssp., agent de la leishmaniose tégumentaire des Guyanas 


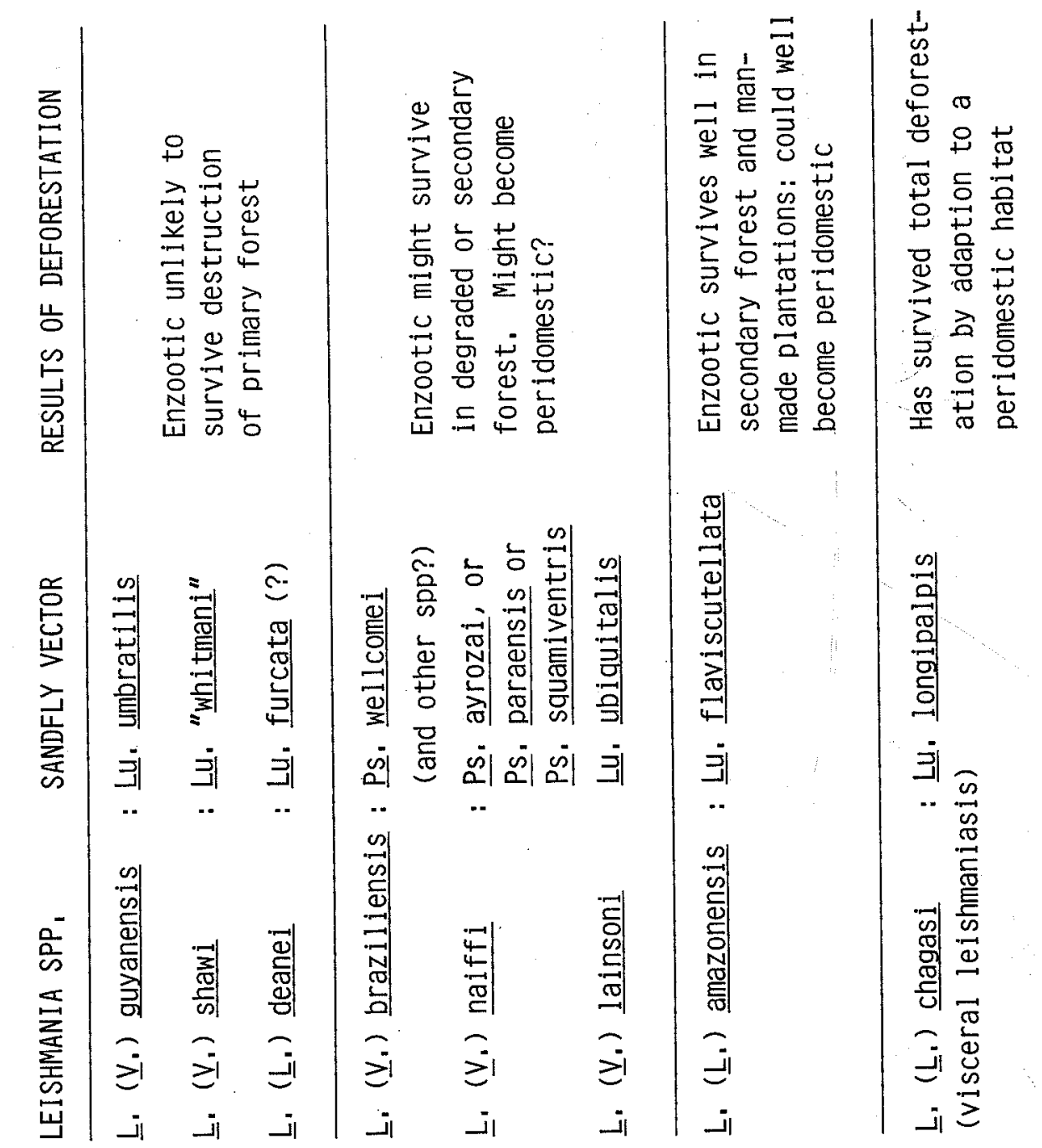


et de l'Amerique Centrale. Arch Inst Pasteur Guy Franc 15: 1-4.

Lainson $\mathbf{R} 1982$. Leishmanial parasites of mammals in relation to human disease, p. 137-179. In MA Edwards \& U McDonnell (eds), Animal disease in relation to animal conservation: Symposia of the Zoological Society of London, 50. Academic Press, London.

Lainson R, Shaw JJ 1972. Leishmaniasis in the New World: taxonomic problems. Brit Med Bull 28: 4448.

Lainson R, Shaw JJ 1977. Leishmanias of neotropical porcupines: Leishmania hertigi deanei nov subsp. Acta Amazónica 7: 51-57.

Lainson R, Shaw JJ 1979. The role of animals in the epidemiology of South American leishmaniasis, 1116 In WHR Lumsden \& R Killick-Kendrick (eds) Biology of the Kinetoplastida Vol 2, Academic Press, London

Lainson R, Shaw JJ 1987. Evolution, classification and geographic distribution, 1-120 In W Peters \& $R$ Killick-Kendrick The Leishmaniases in Biology and Medicine Vol 1 Biology and Epidemiology, Academic Press, London.

Lainson R, Shaw JJ 1989. Leishmania (Viannia) naiffi sp.n. a parasite of the armadillo, Dasypus novemcinctus (L.) in Amazonian Brazil. Ann Parasit Hum Comp 64: 3-9.

Lainson R, Shaw JJ 1992. A brief history of the genus Leishmania (Protozoa: Kinetoplastida) in the Americas, with particular reference to Amazonian Brazil. Ciência e Cultura 44: 94-106.

Lainson R, Braga RR, de Souza AAA, Póvoa MM, Ishikawa EAY, Silveira FT 1989. Leishmania (Viannia) shawi sp.n. a parasite of monkeys, sloths and procyonids in Amazonian Brazil. Ann Parasit Hum Comp 64: 200-207.

Lainson R, Shaw JJ, Braga RR, Ishikawa EAY, de Souza AAA, Silveira FT 1988. Isolation of Leishmania from monkeys in the Amazon Region of Brazil. Trans R Soc Trop Med Hyg 82: 231.

Lainson R, Shaw JJ, Ready PD, Miles MA, Póvoa MM 1981. Leishmaniasis in Brazil XVI. Isolation and identification of Leishmania species from sandflies, wild mammals and man in north Para State, with particular reference to Leishmania braziliensis guyanensis, causative agent of "pian-bois". Trans $R$ Soc Trop Med Hyg 75: 530-536.

Lainson R, Shaw JJ, Silveira FT, Braga RR, Ishikawa EAY 1990. Cutaneous leishmaniasis of man due to Leishmania (Viannia) naiffi Lainson \& Shaw, 1989. Ann Parasitol Hum Comp 65: 282-284.
Lainson R, Shaw JJ, de Souza AAA, Silveira FT, Falqueto A 1992. Further observations on Lutzomyia ubiquitalis (Psychodidae: Phlebotominae), the sandfly vector of Leishmania (Viannia) lainsoni. Mem Inst Oswaldo Cruz 87: 437-439.

Lainson R, Shaw JJ, Ward RD, Ready PD, Naiffi RD 1979. Leishmaniasis in Brazil XIII. Isolation of Leishmania from armadillos (Dasypus novemcinctus), and observations on the epidemiology of cutaneous leishmaniasis in north Para State. Trans $R$ Soc Trop Med Hyg 73: 239-242.

Le Pont F, Pajot F-X 1981. La leishmaniose en Guyane française 2. Modalités de la transmission dans un village forestier: Cacao. Cahiers ORSTOM Sér Ent Méd Parasitol 18: 359-382.

Menezes JA, Brazil RP, Momen H, Grimaldi G 1989. Atypical human leishmaniasis due to a parasite of the Leishmania braziliensis complex in north Brazil. Trans R Soc Trop Med Hyg 83: 429.

Naiff RD, Freitas RA, Arias JR, Barrett TV 1989. Aspectos epidemiológicos de uma Leishmania de tatus (Dasypus novemcinctus) Prog Resum XI Congr Bras Parasitol, p. 24

Saf'janova VM 1982. The problem of taxonomy with Leishmania, p. 5-109. In The Leishmanias, Ser Protozool Soviet Acad Sci. Science (Leningrad) 7: (in Russian).

Shaw JJ, Ishikawa EAY, Lainson R, Braga RR, Silveira FT 1991. Cutaneous leishmaniasis of man due to Leishmania (Viannia) shawi Lainson, de Souza, Póvoa, Ishikawa \& Silveira, in Pará State, Brazil. Ann Parasitol Hum Comp 66: 243-246.

Silveira FT, Lainson R, Shaw JJ, Braga RR, Ishikawa EAY 1991a. Leishmaniose cutânea na Amazônia. Isolamento de Leishmania (Viannia) lainsoni do roedor Agouti paca (Rodentia: Dasyproctida), no Estado do Pará, Brasil. Rev Inst Med Trop S Paulo 33: 18-22.

Silveira FT, Shaw JJ, Braga RR, Ishikawa E 1987. Dermal leishmaniasis in the Amazon Region of Brazil: Leishmania (Viannia) lainsoni sp.n. a new parasite from the State of Pará. Mem Inst Oswaldo Cruz 82: 289-292.

Silveira FT, de Souza AAA, Lainson R, Shaw JJ, Braga RR, Ishikawa EAY 1991b. Cutaneous leishmaniasis in the Amazon region: natural infection of the sandfly Lutzomyia ubiquitalis (Psychodidae: Phlebotominae) by Leishmania (Viannia) lainsoni in Pará State, Brazil. Mem Inst Oswaldo Cruz 86: 127-130.

Vianna G 1911. Sôbre uma nova espécie de Leishmania (Nota preliminar). Bras-Med 25: 411 . 